\title{
Geschlecht, Beziehung, Politik. Welche Möglichkeiten und Grenzen „erfolgreichen“ Handelns hatte Bianca Maria Sforza?
}

\author{
von Christina Lutter
}

Fragestellungen zu Geschlechterrollen und Geschlechterverhältnissen für den Zeitraum der Frühen Neuzeit haben in den vergangenen Jahren eine beeindruckende Forschungsliteratur hervorgebracht. ${ }^{1}$ Umso erstaunlicher ist es, dass zu diesen Themen für den „Hof “ Maximilians I. trotz seiner beiden Ehen mit Maria von Burgund und Bianca Maria Sforza wenig bekannt ist. ${ }^{2}$ Dies dürfte eine Reihe von Gründen haben: Besonders die Geschlechtergeschichte hat wesentlich dazu beigetragen, die Rolle hochadeliger Frauen und Fürstinnen sichtbar zu machen und damit eine Leerstelle der traditionellen politik- und verfassungsgeschichtlichen Geschichtsschreibung zu füllen. ${ }^{3}$ Dass aber gerade diese Personengruppe vergleichsweise spät umfassenderes Interesse gefunden hat, liegt auch daran, dass sich die ältere, biografisch orientierte Frauenforschung primär herausragender Figuren angenommen und dadurch mit dazu beigetragen hat, sie als „Ausnahmefrauen“ zu konstruieren. Gerade in Abgrenzung dazu lag der programmatische Fokus der feministischen Wissenschaft weniger auf einzelnen Personen als auf nicht privilegierten gesellschaftlichen Gruppen.

Etwa gleichzeitig damit führten die Abwendung von der traditionellen Politikgeschichte und die Hinwendung zu sozial- und kulturhistorischen Fragestellungen besonders im deutschsprachigen Raum dazu, dass politikgeschichtlich besetzte Felder lange Zeit aus dem Fokus des Interesses gerieten. Erst ein erweiterter Politikbegriff im Sinn einer Kulturgeschichte des Politischen rückte anstelle des traditionellen Blicks auf „große“ Persönlichkeiten und Ereignisse, verbunden mit einer linearen und kausalen Chronologie, strukturelle Fragen nach Macht und Herrschaft, Diskurs und Repräsentation, Handlungsspielräumen und politischer Praxis ins Zentrum des Interesses. ${ }^{4}$

Bei der Frage nach den Wechselbeziehungen zwischen Geschlecht und Politik in historischer Perspektive geht es also darum, strukturelle, diskursive und praktische Möglich-

1 Einen guten Überblick über die Veränderungen der Fragestellungen von der Frauen- zur Geschlechtergeschichte bieten Opitz, Um-Ordnungen; und Griesebner, Feministische Geschichtswissenschaft; grundlegend WUNDER, „Er ist die Sonn“; für eine frauengeschichtliche Perspektive DubY (Hg.), Geschichte der Frauen, Bd. 3, Frühe Neuzeit.

2 Vgl. Noflatscher/Niederkorn (Hgg.), Innsbrucker Hof, hier 18; dazu Lutter, Zur Repräsentation: Dieser Beitrag ist gleichzeitig mit dem hier publizierten entstanden. Beide Texte ergänzen einander und sollen im Sinn einer Forschungsskizze verschiedene Aspekte des Themas und Möglichkeiten vergleichender Untersuchungen aufzeigen.

3 WUnder, Herrschaft; Forschungsüberblick in programmatischer Perspektive bei RogGe, Nur verkaufte Töchter?, sowie in den Beiträgen in Keller (Hg.), Gynäkokratie, v. a. Schnettger, Weibliche Herrschaft, ebd. (Zugriff vom 28.7.2009).

4 Stollberg-Rilinger, Kulturgeschichte des Politischen; zur Kritik an der traditionellen Politikgeschichte durch Vertreter der Annales bereits in den 20er Jahren des 20. Jahrhunderts vgl. MidDELL/ SAMmLER (Hgg.), Alles Gewordene hat Geschichte. 
keiten und Grenzen des politischen Handelns von Frauen und Männern unter dem Aspekt von Geschlechterordnungen und im Rahmen ihrer jeweiligen Gruppenkulturen herauszuarbeiten. ${ }^{5}$ Herrschaft ausübende Männer und Frauen waren in einer ständisch strukturierten Gesellschaft grundsätzlich immer Beschränkungen unterworfen, die teilweise mit ihrer Geschlechtszugehörigkeit zu tun hatten, teilweise aber auch nicht. Die Konzeptionalisierung von Geschlecht als relationale Kategorie erweist sich besonders deshalb als hilfreich, weil sie in diesem spezifischen Untersuchungsfeld dazu geeignet ist, sowohl die dynastischen Aspekte der Familienzugehörigkeit wie auch jene der unterschiedlichen politisch-rechtlichen Legitimationen von Männern und Frauen in Bezug zueinander zu setzen. ${ }^{6}$

Die spezifisch schlechte Forschungssituation für das höfische Umfeld Maximilians I. dürfte zudem mit der besonderen Konzentration der älteren Forschung auf seine Herrscherpersönlichkeit zusammenhängen. ${ }^{7}$ Erst seit den 1990er Jahren gibt es verstärkt systematische Studien zu den strukturellen Aspekten seiner Politik, zur sozialen Formation seines Hofes und der ihn bestimmenden Gruppen sowie zu seinen politischen Beziehungen in einem vergleichenden europäischen Zusammenhang. ${ }^{8}$ Der Mangel an Untersuchungen zu Geschlechterbeziehungen im Umfeld Maximilians mag darüber hinaus auch ein Effekt der Überlieferungssituation sein. Denn einerseits sind aufgrund der zunehmenden politischen Kommunikation und Verwaltungstätigkeit die Quellen zur Herrschaft Maximilians I. im Vergleich zum Zeitraum davor zahlreicher, andererseits sind sie aber noch deutlich weniger systematisch erhalten als in den Jahrzehnten danach. ${ }^{9}$ Schließlich hat der Befund wohl auch mit den spezifischen personellen Konstellationen und politisch-dynastischen Kräfteverhältnissen in den Jahrzehnten der Herrschaft Maximilians zu tun, in denen seine Verbindungen mit der früh verstorbenen Maria von Burgund und der - im Vergleich mit ihr, aber auch mit seiner Tochter Margarethe als Regentin der habsburgischen Niederlande - wesentlich weniger konturierten Bianca Maria Sforza verortet sind.

Der Fokus dieses Beitrages liegt im Folgenden auf den politischen Konstellationen um die zweite Verbindung Maximilians mit Bianca Maria. ${ }^{10}$ Pointiert formuliert scheint sie sowohl von den Zeitgenossen wie auch von der spärlichen biografischen Forschung zu ihrer Person fast ausschließlich auf ein misslungenes dynastisches Projekt reduziert wahrgenommen worden zu sein. ${ }^{11}$

5 Gerade hier, so der Befund von Rogge, Nur verkaufte Töchter?, und Keller, Editorial zu Dies. (Hg.), Gynäkokratie bzw. ebd. SchnetTger, Weibliche Herrschaft, gibt es aber nach wie vor einen Mangel an vergleichenden Untersuchungen. Matthias Schnettger weist zudem darauf hin, dass die Überbewertung der Möglichkeiten einzelner Herrscherinnen den Blick auf die strukturell bedingten Spielräume, aber auch Grenzen des politischen Handelns von Frauen verstellt hat.

6 Scott, Gender; dazu Wunder, Dynastie.

7 So auch noch Wiesflecker, Maximilian I.; vgl. den Überblick bei Hollegger, Maximilian I. Zu dieser Problematik anhand des lange als „autobiografisch“ bezeichneten Repräsentationswerkes Maximilians: MüLLER, Gedechtnus.

8 Grundlegend Müller, Gedechtnus; vgl. Noflatscher, Räte; Lutter, Politische Kommunikation; jetzt Kocher/Sieber (Hgg.), Maximilians Welt.

9 Vgl. Noflatscher, Quellen, mit einer Zusammenstellung der älteren Forschungsliteratur.

$10 \mathrm{Zu}$ einem Versuch einer geschlechtergeschichtlich orientierten, vergleichenden Einordnung der ersten Ehefrau Maximilians, Marias von Burgund, sowie seiner Tochter Margarethe vgl. LutTER, Repräsentation.

11 Eine Suche nach dem Begriff „Bianca Maria Sforza“ in der Datenbank der Regesta Imperii - Online Quellen zur Reichsgeschichte (RI, Zugriff vom 27.7.2009) ergibt insgesamt 19 Titel zwischen 1875 
Worin ist diese Wahrnehmung begründet und inwieweit lässt sie sich durch geschlechtergeschichtliche Fragestellungen differenzieren? Welche Rollenmodelle und Identifikationsangebote standen Bianca Maria - etwa im Unterschied zu ihrer „erfolgreicheren“ Vorgängerin, Maria von Burgund, aber auch zu ihrer kaum jüngeren Stieftochter Margarethe - zur Verfügung, und welche Handlungsspielräume hatte sie in ihrem konkreten sozialen Umfeld?

Maria und Margarethe repräsentieren unterschiedliche „Idealtypen“ zeitgenössisch erfolgreicher politischer Modellbildung, welche die Gleichzeitigkeit und Unabgeschlossenheit von geschlechtlich markierten Selbstentwürfen und Fremdbildern deutlich machen. ${ }^{12}$ Steht in beiden Fällen der dynastische Aspekt im Vordergrund, wurde er doch unterschiedlich in ihre Lebensentwürfe integriert. Maria repräsentiert aufgrund ihres herausragenden Status als burgundische Erbtochter und Mutter des Thronfolgers ein traditionelles Rollenmodell, das sie infolge ihres frühen Todes kaum modifizieren konnte. Dafür wurde sie genau deshalb in der habsburgischen memoria zur maßgeblichen Figur an der Seite Maximilians. Hingegen konnte Margarethe aus einer dynastisch zunächst schlechteren Ausgangsposition nach dem Tod ihres Bruders Philipp als zweifache Witwe und Regentin der habsburgischen Niederlande verschiedene geschlechtlich markierte und geschlechtsneutrale Elemente frühneuzeitlicher Herrschaft $\mathrm{zu}$ einem neuen aktiven Typus verbinden. ${ }^{13}$

Mein Augenmerk gilt im Folgenden vor allem jenen über die spezifische politische und personelle Konstellation hinaus reichenden vergleichbaren Faktoren, die aus geschlechtergeschichtlicher Perspektive die Bedingungen und Möglichkeiten politischen Handelns, aber auch seine Grenzen markieren. ${ }^{14}$ Gerade der Umstand, dass Bianca Maria Sforza im Unterschied zu Maria von Burgund bereits im zeitgenössischen Verständnis kein „Erfolgsmodell“ abgab, wirft die Frage auf, von welchen Kriterien der politische „Erfolg“ einer frühneuzeitlichen Fürstin bedingt war. „Erfolg“ bzw. „Misserfolg“ als Beschreibungskategorien verweisen auf Erwartungshaltungen der handelnden Personen und ihrer sozialen Umgebung. So können scheinbar individuelle Handlungen in Relation zu den Intentionen der Akteurinnen und Akteure und im Rahmen der jeweiligen politischen, sozialen und kulturellen Bedingungen untersucht werden. Erfolg und Misserfolg sind zwar auf die handelnde Person bezogen, werden aber nur im öffentlichen Raum wirksam, und sind daher geeignet, die Interaktion von Person und sozialem Umfeld zu erhellen.

Welche Quellentypen zur Beziehung zwischen Maximilian und Bianca Maria Sforza geben Auskunft über zeitgenössische Erwartungen an die Römische Königin, sowie über die Nor-

und 2005, davon fünf seit 1995: drei kleinere kunsthistorische Spezialstudien, eine ungedruckte Dissertation zu ausgewählten Briefen zum Tanzgeschehen um Bianca Maria (Andrea Aigner, Graz 2005), und ein Beitrag zu ihrer Brautfahrt (Luigi Conato, 2001). Die ältere Literatur umfasst zwei Monografien: CALVI, Bianca Maria (1888), bietet die vollständige Wiedergabe einiger Gesandtschaftsberichte und ist in den Regesten Maximilians I (RI XIV 1) aufbereitet. Die Darstellung von Hochrinner, Bianca Maria Sforza (1966), wurde weitgehend auf der Materialbasis der Maximilian-Regesten verfasst. Die Ergebnisse sind in die grundlegende Maximilian-Biografie Hermann Wiesfleckers, Maximilian I., eingeflossen. Nach Abschluss dieses Manuskripts ist erschienen: WeIss, Kaiserin.

12 Dazu LutTer, Repräsentation.

13 Dazu besonders die Beiträge in Eichberger (Hg.), Women of Distinction; vgl. auch TAmussino, Margarete von Österreich.

14 Vgl. die methodischen Überlegungen von Rogge, Nur verkaufte Töchter?, 244, und Schnettger, Weibliche Herrschaft, 1, 35. 
men und Diskurse zu höfischen Geschlechterrollen und -beziehungen? Welche Motive, kulturellen Muster und Traditionen kommen in ihnen zum Ausdruck? Welche Hinweise erhalten wir auf die soziale Praxis, z. B. auf die Beziehung zwischen König und Königin, auf ihr Herrschaftshandeln und dessen Bewertung im öffentlichen Raum?

Die Überlieferung ist reichhaltig: Für den Zeitraum von 1493 bis 1504, für den die Quellen zur Geschichte Maximilians vom Regestenprojekt der Regesta Imperii bereits elektronisch erfasst sind, gibt es ca. 650 Einträge zu Bianca Maria, die normative und praxisbezogene, an unterschiedliche Öffentlichkeiten gerichtete und nach unterschiedlichen Darstellungskonventionen geformte Quellen umfassen. Ein großer Teil davon sind Briefe von königlichen Amtsträgern und Gesandtschaftsberichte über den Hof. Dazu kommen zeitgenössische Chroniken und eine vergleichsweise spärliche urkundliche Überlieferung. Den überwiegenden Teil der Überlieferung machen verschiedene Abrechnungen, Verzeichnisse und Verpflegungslisten aus, die Hinweise auf die zeitgenössische Verwaltungspraxis geben und ebenfalls mehrheitlich in Form von Korrespondenzen erhalten sind..$^{15}$

Eine erste Durchsicht der Quellenbelege unter Verwendung eines erweiterten Politikbegriffs ergibt eine Reihe von thematischen Feldern, die sich sowohl chronologisch wie auch funktional differenzieren lassen, einander aber zugleich überlappen: Die Überlieferung der Jahre 1493 und 1494 betrifft zunächst Anbahnung, Verhandlung und Abschluss der Ehe zwischen Maximilian und Bianca Maria, die Festlichkeiten in Mailand, die Reise und Ankunft der Braut in Innsbruck und ihren dortigen Aufenthalt, und die Feiern nach Ankunft des Bräutigams mehr als 10 Wochen später. ${ }^{16}$ Hier finden wir auch Hinweise auf die Bewertung von Braut und Bräutigam sowie auf die an die neue Fürstin gestellten Erwartungen. Konstante Aspekte in der Überlieferung sind der Hofstaat Biancas und seine Integration in den neuen Funktionszusammenhang, die Beziehung zwischen König und Königin im „Binnenverhältnis“, aber auch in der Repräsentation nach außen, sowie die vor allem ab 1496 alles andere dominierenden Fragen von Kosten, Sparmaßnahmen und die damit verbundenen Versuche, „Ordnung“ zu schaffen.

An dieser Konstellation ändert sich in den folgenden Jahren vergleichsweise wenig: Während die Artikulation von „Anfangserwartungen“ an die „neue“ Königin weniger werden, erfolgt ihre Bewertung nunmehr im Rahmen der Nachrichten zur Beziehung des Königspaares. Diese sind ihrerseits zunehmend von den nun insgesamt im Vordergrund stehenden Kosten- und Organisationsfragen geprägt. Einige dieser Aspekte sollen nun genauer betrachtet werden.

Die Eheschließung zwischen Maximilian und Bianca Maria Sforza war ein strategischpolitisches Projekt. Dies war allen Beteiligten und den Zeitgenossen bewusst und entsprach den dynastischen Traditionen wie auch den zeitspezifischen Konventionen. ${ }^{17}$ Auch die erste

15 Für die Jahre von 1504 bis zum Tod Bianca Marias 1510 beziehe ich mich auf die quellenbasierten ungedruckten Dissertationen von Hochrinner, Bianca Maria; GatT, Innsbrucker Hof; und NiEDERKORN, Hof Maximilians I.; sowie auf WiesfLeCKer, Maximilian I. Zur Quellenkritik für die hier interessierenden Fragestellungen vgl. auch Rogge, Nur verkaufte Töchter?, 246-255.

$16 \mathrm{Zu}$ den einzelnen Schritten von Eheanbahnung bis Alltagsleben vgl. die Systematik bei SpIEss, Unterwegs; außerdem PARAVICINI (Hg.), Alltag; ereignisgeschichtlicher Überblick bei WiESFLECKER, Maximilian I., Bd. I, 363-377.

17 Zum Folgenden zusammenfassend und mit weiterführender Literatur die Beiträge in ScHмIDT-voN Rhein (Hg.), Maximilian I., besonders Noflatscher, Maximilian, 31-48 und Heinig, Maximilian, 69-81. 
Verbindung mit Maria von Burgund war eine politische Allianz gewesen, die bei allen Unterschieden durchaus vergleichbare Elemente aufweist. In beiden Fällen erhofften sich die Habsburger durch die Verbindung mit einer Partnerin von dynastisch zwar niedrigerem Rang, aber herausragenden Ressourcen territoriale und ökonomische Gewinne, während die Familien der jeweiligen Braut eine Statuserhöhung durch die Verbindung mit dem Thronfolger bzw. König des Hl. Römischen Reichs anstrebten. In beiden Fällen ging es zudem um eine wechselseitige Absicherung der Erbfolge. In beiden Fällen war der Ausgang des mit dem jeweiligen dynastischen Projekt verbundenen territorial-militärischen Unternehmens ungewiss. ${ }^{18}$ Bei der Verbindung mit den Sforza war das Gefälle zwischen den Brautleuten in Hinblick auf den Stand ihrer Familien zwar so groß, dass Maximilian den Tod seines standesbewussten Vaters abwartete, bevor die Heiratsverhandlungen begannen, dafür sprengte aber auch die Mitgift der Braut mit 400.000 Gulden weitaus den Rahmen des Üblichen. ${ }^{19}$

Dass die Hochzeit Ende November 1493 mit großem Aufwand zunächst in Abwesenheit des Bräutigams per procuram in Mailand gefeiert wurde, entsprach den Gepflogenheiten. ${ }^{20}$ Der Aufbruch der Braut nach Innsbruck erfolgte nur wenige Tage nach den Feierlichkeiten mit einem repräsentativen Gefolge von 600 Pferden. Bianca selbst und die beiden mailändischen Gesandten Erasmo Brascha und Maffeo Pirovano berichteten täglich von der beschwerlichen und gefährlichen Reise über den Comer See, durch das Veltlin und schließlich über das Wormser Joch, bis man nach knapp drei Wochen am 22. Dezember in Innsbruck eintraf. ${ }^{21}$

Die Königin, so eine erste Bewertung durch Brascha, halte sich gut auf der mühevollen Reise, welche wie die Ankunft in Innsbruck den Ansprüchen an einen repräsentativen Hochzeitszug entsprach: Die Grafen von Matsch, von Baden und von Zollern sowie der Bischof von Chur kamen der Braut mit 100 Pferden entgegen. In Innsbruck wurden die Mailänder von Herzog Sigmund und seiner 40 Jahre jüngeren Ehefrau Katharina von Sachsen feierlich empfangen, die 650 Personen des Hofgesindes der Königin gut untergebracht und verpflegt. Wie es sich gehörte, demonstrierten die Familien beider Partner ihre Bedeutung und ihre Ressourcen. ${ }^{22}$ Sigmund und Katharina organisierten prächtige Feste mit Tanz, Turnieren und Jagden, und die Königin wurde mit großem Wohlwollen betrachtet: Sie habe, so der Sekretär Oswald von Hausen an Zyprian von Serntein, eine schöne Gestalt, und ein klain subtil angesicht. Sie tanze fröhlich, gefalle jedermann, und werde hoffentlich eine fruchtpare kunigin und bey der ku. Mt. schone fursten und hern von Osterreich geperen. Hier finden wir einige der wesentlichen Wahrnehmungs- und Bewertungskriterien für eine fürstliche Braut - Schönheit, höfisches Benehmen sowie Gebärfähigkeit -, denen Bianca in den Augen der Zeitgenossen zu diesem Zeitpunkt offensichtlich entsprach. ${ }^{23}$

$18 \mathrm{Zu}$ den politisch-militärischen Details vgl. ebd. sowie Wiesflecker, Maximilian I., Bd. I, Kap. 4 und 11.

19 Ausführliche Belege zu den Heiratsverhandlungen sowie Details der vertraglichen Vereinbarungen finden sich in RI XIV 1 zum Jahr 1493.

20 Vgl. SpIEss, Unterwegs, mit zahlreichen Vergleichsbeispielen.

21 RI XIV zum Dezember 1493, hier besonders nn. 2868 (15.12.1493), 2874 (19.12.1493), 2875 (20.12.1493); ausführliche Darstellung auch bei Hochrinner, Bianca Maria, 33-40.

22 RI XIV n. 2875 (20.12.1493) zur Bewertung Biancas; weiters nn. 288 und 289 (5.1.1494); vgl. KöFLeR/CARAmelle, Frauen; zur Bedeutung des Hochzeitszuges und des Adventus vgl. hier Spiess, Unterwegs, 28 f.; sowie grundsätzlich JoHANEK (Hg.), Adventus.

23 Zitate RI XIV n. 2878 (24.12.1493) und n. 2898 (13.1.1494); vgl. SPIESs, Unterwegs, 24. 
Als unüblich kommentiert wurde hingegen die wochenlange Abwesenheit des Bräutigams: Maximilian, nach dem Tod Friedrichs III 1493 verantwortlich für die gesamte Reichsund erbländische Politik, wurde in Wien aufgehalten und verschob Zeitpunkt und Ort der Hochzeit mehrfach. Am 9. März 1494 traf das Paar schließlich in Hall zusammen. Nach dem üblichen Zeremoniell mit Tafel und Festlichkeiten zog sich der König dann nochmals bis in die frühen Morgenstunden mit Gesandten und Beratern zurück. Dann wurde das Beilager vollzogen, ad confussione de li inimici nostri, wie Erasmo Brascha nach Mailand berichtet. $^{24}$

Auch die Berichte über die Innsbrucker Hochzeitsfeierlichkeiten und die Beziehung des Königspaares in den folgenden Wochen korrespondieren mit den politischen Erwartungen an die Ehe und enthalten die wesentlichen formalen Elemente ebenso wie die entsprechenden Zeichen öffentlich inszenierter Liebe. Darin unterscheiden sich die Darstellungen nicht wesentlich von jenen über die erste Ehe Maximilians mit Maria von Burgund. Am Sonntag nach dem Beilager trug Bianca beim gemeinsamen Kirchgang die Krone im Wert von 60.000 Gulden, ein Geschenk Maximilians. Danach gab es eine große Tafel, im Anschluss daran hielt der Mailänder Jason Maynus eine feierliche Rede. ${ }^{25}$ Der Ehevertrag wurde ratifiziert, Bianca erhielt, wie Maximilians Mutter Eleonore von Portugal, als Dos Leoben, Weitra, Judenburg und Pordenone mit allem Zubehör. ${ }^{26}$ Maximilian ist liebevoll und überhäuft Bianca mit Geschenken, sie berichtet dem Onkel in Mailand von seiner täglich wachsenden Liebe zu ihr. Die frisch Vermählten verbringen die Nächte miteinander, untertags spielen sie Karten und gehen auf die Jagd, am Abend gibt es Tanzfeste. ${ }^{27}$

Neben diesen Artikulationen gemeinsamer Interessen und herrscherlicher Repräsentation tritt die Römische Königin auch politisch im Sinn des gemeinsamen Herrschaftshandelns auf, so etwa im April 1494, als sie die preces primariae an die Stadt Rothenburg ob der Tauber wegen einer Pfründe für einen Augsburger Kleriker äußert, oder auch beim Empfang verschiedener Gesandtschaften. ${ }^{28}$ Das Paar unternimmt die erste gemeinsame Reise und verbringt einige Monate in den Niederlanden, wo Bianca Maximilians Tochter Margarethe kennenlernt. Auch später gibt es eine Reihe von Hinweisen auf Kontakte zwischen den beiden. ${ }^{29}$ Bereits im Juni 1494 berichtet Biancas Hofmeister Niklas von Firmian, sie sei unzweifelhaft schwanger, eine Hoffnung, die sich jedoch weder damals noch in den folgenden Jahren erfüllte. Immer wieder gibt es Gerüchte über Schwangerschaften, etwa dokumentiert beim venezianischen Diaristen Marino Sanudo, der bald aber auch zu berichten weiß, dass dies gar nicht möglich sei, da sich der König nie bei Bianca aufhalte. ${ }^{30}$

Ähnliches steht Ende November 1494 in einem Brief des Mailänder Gesandten Maffeo Pirovano an Ludovico Moro. Das Schreiben beruht auf einem Memoriale des zweiten mailändischen Gesandten Erasmo Brascha, in dem er seinem Kollegen wichtige Informationen für

24 RI XIV nn. 457, 459, 460 (hier das Zitat) zum 9./10.3.1494; vgl. SpIEss, Unterwegs, 32.

25 RI XIV nn. 477, 478, 480 zum 15./16.3.1494.

$26 \quad$ RI XIV n. 457, um den 14./15.3.1494.

27 RI XIV nn. 461, 477, 491 vom März 1494.

28 Z. B. RI XIV n. 2988 (19.4.1494) für den Augsburger Kleriker Leonhard Lang; z. B. RI XIV n. 8819 (4.9.1498) zur Entscheidung über ein standesgemäßes Geschenk für den polnischen Gesandten.

29 Noflatscher, Maximilian, 36.

$30 \quad$ RI XIV n. 7697 (2.12.1496) nach Marino Sanudo, Diarii I, 409. 
Ludovico auflistet. ${ }^{31}$ Neben diversen persönlichen Anliegen präsentieren diese Notizen eine bemerkenswerte Innensicht der Vorgänge am königlichen Hof und Hinweise auf Erwartungshaltungen gegenüber Bianca. Die Differenz zu den Berichten wenige Monate zuvor ist augenfällig: Die Königin sei nicht sehr klug, und Brascha müsse dementsprechend immer wieder unter vier Augen mit ihr reden, um sie bei Laune zu halten. Manchmal esse sie in ihrem Zimmer auf den Knien und müsse von Brascha und Pirovano ermahnt werden. Maximilian gegenüber aber verhalte sie sich - entgegen anders lautender Berichte - würdig und sei von einzigartiger Bescheidenheit. ${ }^{32}$

Maximilian selbst sei viel unterwegs, sehr beschäftigt und besuche die Königin nie vor dem Schlafengehen. Er liebe sie aus ehelichem Pflichtgefühl. Er habe mit den Ärzten über Biancas Verdauungsprobleme gesprochen, welche eine Empfängnis verhinderten. Hier wird also bereits in der zentralen Frage der fehlenden Nachkommenschaft die „Schuld“ tendenziell in Richtung Königin verschoben. Im Vergleich mit seiner ersten Ehefrau, so Maximilian grundsätzlich, verfüge Bianca zwar über ähnliche Schönheit und Freigiebigkeit, „die andere (l'altra) hatte aber mehr Erfahrung; er hoffe, diese (questa) werde sich noch machen“. ${ }^{33}$

Neben diesen Einblicken in den Beziehungsalltag des Paares berichten die Gesandten auch über Konflikte bei Hof: Biancas Hofstaat befinde sich in Unordnung, die „Deutschen“ beschuldigten die „Lombarden“ der Verschwendung, und besonders der Hofmeister Niklas von Firmian behandle sie schlecht. Einige der Mailänder Hofleute werden wegen ihrer besonderen Treue zum Herzog gelobt, andere hingegen als wenig vertrauenswürdig eingestuft, weil sie zum „Haus“ der Herzogin Bona gehörten. ${ }^{34}$ Das Hauptproblem - und das bestätigen die Äußerungen der Hofleute Maximilians - stellte aber das Ehepaar Pietro Caimo und Violanta Caima dar. Violanta dürfte damals die engste Vertraute Biancas gewesen sein. Sie und ihr Mann standen der Königin nach der Nachricht vom Tod ihres Bruders Gian Galeazzo bei, infolge dessen Ludovico „il Moro“ Sforza, der Onkel der Geschwister, auch formal die Herrschaft im Herzogtum Mailand übernehmen konnte. ${ }^{35}$ Violanta habe sich, so Brascha, anläßlich der Todesnachricht sehr verdächtig betragen, Bianca hingegen unterstütze den Onkel. Die Hintergründe der offenbar vielfältigen Konflikte sind aus den oft widersprüchlichen Äuße-

31 Petitione et desiderii particulari de Messer Herasmo, quali se hanno ad intercedere cum lo Illustrissimo Signore Duca de Milano, per mezo de la Illustrissima Duchessa, ediert bei Calvi, Bianca Maria, Documento VI, 155-161; dort auch ein zweites memoriale, welches offenbar als Instruktion für ein weiteres Schreiben an die Mailänder Herzogin Beatrice d'Este gedacht war. Edition des Briefes des Pirovano und Interpretation dieser beiden Texte ebd. 82-89 und 89-93; vgl. auch RI XIV n. 1162 vom 23.11.1494, Antwerpen.

$32 \quad$ Ebd., 156 und 157.

33 Ebd., 157: Del stare del re absente 20 di dala Regina, et che la Maestà sua sta tutto el di in facende, et non va mai dala regina più presto che alora del dormire. Chel Re ama epsa Regina per honore et debito del matrimonio. De le parole usate per lo prefato Re cum li medici sopra la vita de la Regina, et sopra la indigestione, la quale prohibiva la conceptione, et se non fusse stato per lo caso del Duca, la Maestà sua era per darli remedio. De la comparatione facta per lo Re de le mogliere sue, dicendo che questo assimilia all'altra de beleza et de liberalità, ma che l'altra era di più experientia, et che sperava ancora che questa se faria. Ähnlich, wenn auch weniger persönlich, auch spätere Berichte, z. B. Angelo da Fiorenza nach Mailand: RI XIV n. 2465 vom 22.9.1495, Worms.

35 Dementsprechend hielten sich Gerüchte, dass Gian Galeazzo keines natürlichen Todes gestorben sei. Zusammenfassung der politischen Hintergründe bei Hollegger, Maximilian I., 90-94. 
rungen kaum eruierbar. Violanta wird von Brascha eine Allianz mit dem Hofmeister und der Hofmeisterin Niklas und Paula von Firmian vorgeworfen, aber auch pratiche mit dem neapolitanischen Gesandten Hieronimo Vento, worauf Maximilian der Königin weitere Audienzen mit diesem untersagte. Außerdem ging es um Rangstreitigkeiten: Pietro Caimo kümmere sich nicht um die Befehle des Hofmeisters und streite laut mit dem Vorschneider, an dessen Tisch er bei den Mahlzeiten nicht sitzen wolle. Brascha setzte sich mehrfach für die Abberufung des Paares ein; auch der Bischof von Mainz habe bereits dafür interveniert und Maximilian selbst wünsche ihre Rückkehr nach Mailand. ${ }^{36}$

Violanta und Pietro wehrten sich geraume Zeit erfolgreich gegen eine Abberufung. Noch zwei Jahre später sind beide in der königlichen Umgebung nachweisbar, abermals geht es um Intrigen zwischen den lombardischen und anderen Hofparteien, die gleichsam die mikropolitische Dimension der politischen Auseinandersetzungen um das Herzogtum Mailand und die Spitze des Eisbergs der höfischen Integrationsprobleme darstellen. Trotz weiterer Interventionsversuche Violantas bei Maximilian im Herbst 1496 wurden die beiden schließlich entlassen. ${ }^{37}$

Etwa gleichzeitig mit den Nachrichten der mailändischen Gesandten in der zweiten Jahreshälfte 1494 äußerte auch der Hofmeister der Römischen Königin Probleme wegen der fehlenden Ordnung ihres Hofstaates. ${ }^{38}$ Die Situation verschärfte sich eklatant aufgrund der Budgetknappheit im Kriegsjahr 1496 und der damit verbundenen Versorgungsengpässe. Ende Mai ersuchten die königlichen Räte Maximilian dringend um Überweisung von Geld nach Worms, weil die Mittel für die speisung nur noch für drei bis vier Tage reichen würden. Ende Juli berichteten sie, dass die Lieferungen eingestellt und Kredite verweigert würden und einige den Hof bereits aus Hunger verlassen hätten. Spott und üble Nachrede seien nicht mehr zu verhindern. ${ }^{39}$ Im August instruierte Maximilian die Schatzkammer in Innsbruck mehrfach, 9.000 Gulden für die Auslösung der Königin und ihres Hofes in Worms aufzubringen, der gleichzeitig aus diesem Anlass geordnet und verkleinert werden sollte. ${ }^{40}$ Vom 3. Oktober 1496 datiert ein langes Schreiben des Niklas von Firmian an den König, in dem er sich über die Missstände bei Hof beschwert und von einem ersten „Ordnungsversuch“ berichtet:41 Wieder geht es um die drastischen Versorgungsschwierigkeiten, um die Probleme mit dem „Welschen“ Hofstaat und um Meinungsverschiedenheiten mit der Königin. Wieder ist von Violanta Caima die Rede, aber auch von einem unliebsamen Stallmeister und einem Küchenmeister, der zuviel verbraucht. 1497 fand dann tatsächlich in Firmians Sinn die weitgehende Auflösung des mailändischen Hofstaates Biancas statt; weitere Abfertigungen von Hofdamen erfolgten $1500 .^{42}$

36 Vgl. die Notizen Braschas bei Calvi, Bianca Maria, 158, sowie dessen Briefe an Maffeo Pirovano vom 11.12.1494 (RI XIV n. 1217) und an Herzog Ludovico vom 17.12.1494 (RI XIV n. 1236). Ausführlich bei Niederkorn, Hof Maximilians, 37-45.

37 Vgl. RI XIV nn. 7429 (24.9.1496), 7473 (3.10.1496), 7543 (20.10.1496), 7656 f. (beide 24.11.1496), 7696 (2.12.1496).

38 Vgl. Firmian an Paul von Liechtenstein bereits am 25.6.1494 (RI XIV n. 824); sowie Firmian an Serntein vom 13. oder 20.11.1494 (RI XIV n. 1160).

$39 \quad$ RI XIV n. 7024 (27.5.1496), n. 7194 (24.7.1496).

40 RI XIV n. 4210 (vor dem 14.8.1496) und n. 4211 (zwischen dem 14.8. und dem 27.8.1496).

$41 \quad$ RI XIV n. 7473.

42 RI XIV nn. 8265, 8302, 8380, 5644; sowie RI XIV n. 14421. 
Das Schreiben Firmians und viele weitere seiner Art dokumentieren ein Strukturelement maximilianeischer Politik. Statt umfassender Ordnungen im normativen Sinn verfügte der König Ordnungsmaßnahmen mit sachlicher, zeitlicher und räumlicher Beschränkung, die aktuelle Missstände beheben sollten. ${ }^{43}$ Wie die Maßnahmen des Jahres 1496 für den Hofstaat der Königin zeigen, war der Sparzwang zentrale Motivation für möglichst effektives Ordnungshandeln. Ein stat (oder état) sollte zunächst einen Überblick über die tatsächlichen Kosten schaffen. Bei allen erhaltenen Ordnungsversuchen für den Hofstaat Biancas handelt es sich um solche Übersichtslisten, die zudem oft nur Teilbereiche des Geschehens bei Hof betrafen, so etwa Tafellisten, Aufstellungen der Kleidung der Königin und ihrer Hofdamen oder Verpflegungslisten, die auch das Gesinde umfassen. ${ }^{44}$ Sie überliefern daher auch recht unterschiedliche Größenordnungen, da teilweise nur der engere Kreis der Frauen um die Königin, teilweise auch der erweiterte Hof inklusive des männlichen und weiblichen Gesindes erfasst wurde und beide nicht zuletzt aufgrund der Sparmaßnahmen einer starken Fluktuation unterworfen waren. ${ }^{45}$

„Ordnungsversuche“ im Sinn von normativen Instruktionen für das „Frauenzimmer“ in Innsbruck gab es hingegen davor und danach: ${ }^{46} 1483$ erließ Herzog Sigmund von Tirol im Vorfeld seiner Hochzeit mit Katharina von Sachsen eine solche Instruktion für die höfische Umgebung seiner zweiten Ehefrau, für die er die pesten und hübschisten Mädchen suchte. ${ }^{47}$ Die nächste derartige Ordnung aus dem Jahr 1519 betraf den Hofstaat der jungen Fürstinnen Maria, Schwester Karls (V.) und Ferdinands (I.), und Anna, Ehefrau des letzteren, die nach der „Wiener Doppelhochzeit“ von 1515 in Innsbruck residierten. Beide Ordnungen geben einen guten Einblick in die disziplinierenden Vorstellungen vom Leben im engeren und weiteren Umfeld der Königin bzw. Fürstin, die in dieser Form wohl spezifisch „Frauenräume“ betrafen. Die Aufgaben von Hofmeister und Hofmeisterin sind ebenso klar reglementiert wie die Zusammensetzung des Frauenzimmers, der Tagesablauf der Hofdamen und die restriktiv gehaltenen Kontakte nach außen.

Diese Texte erzählen viel über die Idealvorstellungen zum Alltag im Frauenzimmer, aber wenig über seine Entsprechung in der tatsächlichen Praxis. So gibt es immer wieder Berichte vom lustigen Leben im Frauenzimmer der Königin. ${ }^{48}$ Am bekanntesten ist das Beispiel der Apollonia Lang, der Schwester des einflussreichen königlichen Sekretärs Matthäus Lang. Sie

43 Vgl. die Beiträge in Kruse/Paravicini (Hgg.), Höfe, besonders Heinig, Theorie, hier 225-229 und 241 f., außerdem Noflatscher, Quellen.

44 Die wichtigsten Ordnungsversuche finden sich zusammengestellt bei HeInIG, Theorie, und NofLATSCHER, Quellen, 34 f. Der stat von 1506, eine Liste von Personen im engeren Umfeld der Königin, ist bei Niederkorn, Hof Maximilians I., 349 f. ediert; vgl. auch Hochrinner, Bianca Maria, 110118.

45 RI XIV n. 15368 (19.5.1501) erschließt einen Gesamtumfang von 200-230 Personen. Im Vertrag mit Georg Gossembrot vom 28.8.1501, in dem ihm Maximilian die gesamten Einnahmen der oberösterreichischen Länder verpfändete, ist von 110 Personen und 60 Pferden die Rede, die mit 9.000 Gulden unterhalten werden sollten (RI XIV n. 12393); dieser Betrag wurde später auf 13.000 Gulden erhöht (RI XIV n. 17975 vom Dezember 1503). Eine Verpflegungsliste des Hofstaats Biancas vom 8.6.1504 nennt 154 Personen (RI XIV n. 18846).

46 Vgl. dazu die Beiträge in Hirschbiegel, (Hg.), Frauenzimmer, besonders Heinig, Zucht und ordnung, und Bojcov, Frauenzimmer.

47 Zitat bei Bojcov, Frauenzimmer, 202.

48 z. B. RI XIV nn. 1130 (5.11.1494), 1705 (14.5.1495), 3663 (21.12.1495). 
war geraume Zeit die Geliebte Herzog Georgs von Bayern, der sich daher mit seinem Gefolge häufig am Hof Biancas aufhielt. Bemerkenswert ist etwa der Bericht der Augsburger Chronik zum Reichstag 1500 über den Einzug des Königspaares, wonach Apollonia Lang am prächtigsten von allen gekleidet war, da sich der Bayernherzog das leisten konnte. ${ }^{49}$ Gemeinsam mit Niklas von Firmian bemühte sich Matthäus Lang aber schon ab 1496 um eine „ehrliche“ Verheiratung seiner Schwester, was schließlich mit dem Grafen von Lodron auch gelang. 1503 wurde ihre Hochzeit besonders prunkvoll in Anwesenheit des Königspaares und des Thronfolgers Philipp gefeiert. ${ }^{50}$ Aber auch Lang selbst hatte offenbar eine junckfrawe im Frauenzimmer der Königin, und von Maximilians Protonotar Zyprian von Serntein gibt es gleich mehrere Berichte über seine Vorliebe für das Frauenzimmer. ${ }^{51}$

Derlei Nachrichten und das Fehlen formaler Ordnungen bedeuten jedoch nicht, dass der König deshalb weniger Eingriffe in den Hofstaat seiner Gemahlin tätigte. Im Gegenteil: aus allen Maßnahmen ab 1496 geht klar hervor, dass er der oberste Herr des Personenverbandes um die Königin war. Besonders eindrucksvoll verdeutlicht dieses Selbstverständnis ein Schreiben Maximilians an Friedrich von Sachsen, der ihn nach dem Tod Herzog Sigmunds 1496 und der Wiederverheiratung Katharinas, Friedrichs Tochter, gebeten hatte, deren Frauenzimmer in Biancas Hofstaat zu übernehmen. ${ }^{52}$ Der König kam dieser Bitte nur ungern nach, denn es sei üblich, dass beim Tod des Fürsten oder der Fürstin die hofjunckfrawen mit einer entsprechenden Abfertigung verabschiedet würden. Nun aber habe sich die schlechte Sitte eingestellt, dass diese so gern bei Hof lebten, dass sie nicht heiraten wollten, sodass man sie nit als hofjunckfrawen sonder wol hof alt frawn underhalten mues, das dan wider all hofs ordnung zier und frewd ist und nemlich ain gancze widerwertikait. ${ }^{53}$ Dies habe mehrere Gründe, so Maximilian: Erstens hätten die Jungfrauen nach einiger Zeit bei Hof kein Interesse an einem eigenen hewslichen wesen oder hawshalten. Zweitens würden sie sich in Personen verlieben, die nicht für eine standesgemäße Heirat geeignet seien. Sie schlügen einen jarmarkt oft im herczen auf im Glauben, mit der Zeit würden sie nach ihrer Neigung heiraten, und bedächten nicht, dass derlei nicht einer unter zehn widerfährt. All das käme auss uncaischen grunt. ${ }^{54}$ Drittens würden sie von den alten hofjunckfrawen zu solchem Verhalten verführt, damit diese nicht ihre Gespielinnen verlören und allein veralten und weil sie, da sy junk und hupss sein, viel bessere Gesellschaft zur täglichen Kurzweil ins Frauenzimmer zögen.

Deshalb könnten die Jungfrauen schließlich nicht mehr ordentlich verheiratet werden, weshalb er, Maximilian, und auch ain jeder furst ytzo vil junckfrawen in ir frawenzimmer zunemen allain aus den vorgemelten ursachen scheuchen. Zwar könne er einige der Jungfrauen Katharinas für zwei Jahre in das Frauenzimmer Biancas übernehmen. Sollten sie danach nicht verheiratet sein, werde er sich nicht mehr für sie verantwortlich fühlen, sondern sie haimerlauben. ${ }^{55}$

49 RI XIV n. 10071 (8.4.1500) nach Sender, Augsburger Chronik IV, 79.

50 RI XIV n. 7691 (1.12.1496); RI XIV nn. 17695, 17706, 17711, 17865 zu den Hochzeitsfeierlichkeiten im Oktober 1503.

51 RI XIV n. 7242a (8.8.1496) zu Matthäus Lang; zu Zyprian von Serntein z. B. RI XIV n. 7049 (3.6.1496), n. 9008 (13.1.1499).

52 Ediert bei Kraus, Beziehungen, 56-58; vgl. Bojcov, Frauenzimmer, $328 \mathrm{f}$.

53 Kraus, Beziehungen, 56.

54 Ebd., 57: der Begriff für „verlieben“ lautet sbaeczliebe, Spassliebe.

55 Ebd., 57 und 58; vgl. dazu RI XIV n. 7211, Schreiben der kgl. Räte in Innsbruck vom 29.7.1496 über die Schwierigkeiten, den Hofstaat Sigmunds und Katharinas mit 74 Personen und 33 Pferden weiter 
In dieser „Problemanalyse“ kommen ein patriarchales Selbstverständnis und geschlechtlich differenzierte moralische Vorstellungen ebenso zum Ausdruck, wie sie auf eine umfassende Interventions- und Klientelpolitik verweist. Dies belegen die zahlreichen Beispiele zu Maximilians Praxis der Anstellung, Ausweisung, Verheiratung und sonstigen Versorgung des Personals seiner Gemahlin. Als „Herr des Hauses“ und Familienoberhaupt verheiratete er Hofdamen wie Höflinge und versorgte ihm nahe stehende Personen mit günstigen Verbindungen. ${ }^{56}$ Er griff auch in den Hofstaat seiner erwachsenen Kinder Philipp und Margarethe ein. Wechselseitige Empfehlungen von Hofleuten spielen eine prominente Rolle im Briefwechsel zwischen ihm und seiner Tochter. Die traditionell ständischen Vorstellungen von Schutz bzw. Protektion einerseits, und Verfügungsgewalt über die eigenen Leute andererseits waren für beide ungeachtet des Geschlechts der Betroffenen selbstverständlich und Teil einer zeitspezifischen „Sozialpolitik“ im Personenverband. ${ }^{57}$

Auffallend ist aber, welch geringe Rolle Bianca bei all diesen Maßnahmen spielt. Zwar treten König und Königin auch in den späteren Jahren ihrer Beziehung immer wieder gemeinsam bei den von Maximilian angebahnten Hochzeiten oder der Taufe von Kindern auf, gelegentlich ist auch von der Zustimmung Biancas zu Heiratsvereinbarungen die Rede. ${ }^{58}$ Als eigenständig Handelnde wird die Königin dabei nur selten sichtbar, im Unterschied zu ihrer Stieftochter Margarethe, mit der Maximilian handfeste Auseinandersetzungen um ihr Personal hatte. ${ }^{59}$ Allerdings informieren die Quellen vom Hof sehr wohl über Beschwerden der Königin angesichts der immer drastischeren Sparmaßnahmen, die offensichtlich speziell auf dem Rücken ihres Hofstaates ausgetragen wurden. In einer Reaktion auf Biancas Widerstand gegen die Einsparungen durch den Gossembrot-Vertrag modifiziert Maximilian seine Anweisungen an den Innsbrucker Hofrat: Ein Kaplan wird der Königin entzogen, der zweite dem Grafen von Anhalt zugeteilt; den „welschen“ Schneider darf sie behalten, ebenso den Apotheker und einen Edelknaben, die anderen sind zum Kriegsvolk abzustellen. Das Frauenzimmer soll verkleinert und ebenso die Anzahl der Pferde verringert werden. Die Hofnärrin soll gut behandelt und versorgt werden. ${ }^{60}$

Trotz aller Sparmaßnahmen wurde Biancas Hofstaat immer wieder verpfändet, oft reichte das Geld nicht einmal für Winterkleidung. Im Widerspruch zu allen Verschwendungsargumenten fällt dabei auf, dass die Summen, die der Römischen Königin und ihrem Hof zur Verfügung stand, deutlich geringer waren als jene für den König selbst, aber auch für Katharina und Sigmund und später die Prinzessinnen Maria und Anna. ${ }^{61}$

Auch die Häufigkeit des Zusammenseins von König und Königin nahm in der zweiten Hälfte ihrer Beziehung deutlich ab. Waren sie von 1494 bis 1502 noch insgesamt 3,5 Jahre zusammen, so reduzierten sich die gemeinsamen Aufenthalte von 1502 bis zu Biancas Tod

zu unterhalten. Die kriegstauglichen Männer ebenso wie jene aus dem Gefolge Biancas sollten laut einer Instruktion des Königs vom August 1496 nach Mailand geschickt werden (RI XIV n. 4211). Z. B. RI XIV n. 846 (30.6.1494), n. 13533 (14.7.1499), n. 16464 (13.5.1502).

Viele Beispiele bei LE GLAY (Hg.), Correspondance.

Z. B. Zustimmung Bianca Marias zur Heirat zwischen Christoph von Scherffenberg und der Magdalena von Casstelalt aus ihrem Frauenzimmer: RI XIV n. 12363 (20.8.1500).

Noflatscher, Maximilian, 42.

RI XIV n. 16435 (7.5.1502).

Belege bei Hochrinner, Bianca Maria, 85-92; zu den Budgets im Vergleich siehe Wiesflecker, Kammerraitbücher, 93-100. 
1510 auf 1,5 Jahre. ${ }^{62}$ Dies hat mit der ununterbrochenen politischen und militärischen Reisetätigkeit Maximilians ebenso zu tun wie mit dem Aufwand und den Kosten des Reisens mit großem Gepäck. Prunkvolle gemeinsame Auftritte gab es zwar auch noch nach 1500, so etwa in diesem Jahr beim Augsburger Reichstag oder 1503 anlässlich der Hochzeit von Apollonia Lang. 1506 erfolgte eine längere gemeinsame Reise durch Innerösterreich. ${ }^{63}$ Insgesamt überwiegen aber bereits ab 1496 jene Nachrichten, welche die Königin ohne Geld und Bewegungsspielraum zeigen: Immer wieder schreibt sie Maximilian oder lässt ihm durch Leute seines Vertrauens ausrichten, er möge doch zu ihr kommen oder sie zu sich holen, sie wäre nirgends lieber als bei ihm. Dieser Eindruck wird auch durch die regelmäßigen Berichte ihres Hofmeisters verstärkt, die Königin sei traurig und es wäre gut, wenn Maximilian sie besuchen würde. ${ }^{64}$

Welche Faktoren lassen sich nun als ausschlaggebend für dieses Gesamtbild ausmachen? Im Vergleich mit Maria von Burgund ist das dynastische „Defizit“ Biancas, die fehlende Nachkommenschaft, sicher ein wesentliches Moment. Dazu kommt die politisch erfolgreiche Integration der habsburgischen und burgundischen Länder bei gleichzeitiger Verklärung der Person Marias durch ihren frühen Tod, während das italienische Unternehmen Maximilians scheiterte und Bianca gleichsam „ohne Auftrag“ überlebte. Ob und inwieweit es Maria in ihrer Rolle als Ehefrau Maximilians längerfristig gelungen wäre, eine eigenständige Politik wie etwa ihre Tochter Margarethe zu entwickeln, muss offen bleiben.

$\mathrm{Zu}$ den pragmatischen Gründen des „Misserfolges“ Biancas gehört auch das Scheitern der Integration ihres Hofes, die vor allem durch die politische Konstellation der Jahre um 1500 verunmöglicht wurde. Gelang es anderen Fürstinnen, die unterschiedlichen Gruppen bei Hof durch eine systematische Heirats- und Klientelpolitik zu integrieren ${ }^{65}$, scheint dies hier unerreichbar gewesen zu sein. Dabei erscheinen die Konflikte zwischen einzelnen Personen und Gruppen sowie die wechselseitigen Fremdwahrnehmungen von „Welschen“ und „Deutschen“ nur als Spitze des Eisbergs. Maßgeblicher dürfte neben dem politisch bedingten Misstrauen die spezifische Regierungssituation des Römischen Königs zwischen Reichspolitik und Zuständigkeiten in seinen Erbländern gewesen sein. Das „Maximalprogramm“ des Königs hatte zur Folge, dass die budgetäre und verwaltungstechnische Wirklichkeit mit dem rein geografischen Umfang seines Herrschaftsgebietes und seinen Ansprüchen in keiner Weise mithalten konnte. ${ }^{66}$

Margarethe erhielt nach dem Tod ihres Bruders, des Thronfolgers Philipp, mit den habsburgischen Niederlanden einen Verantwortungsbereich übertragen, den ihr Vater selbst nicht mehr zusätzlich übernehmen konnte. Ihre Erziehung in Frankreich und am burgundischen Hof und ihre damals bereits weit reichende politische Erfahrung schufen dabei Spielräume, die sie als Regentin auch zu nutzen verstand, wohl nicht zuletzt deshalb, weil sich die Eingriffe Maximilians schon aufgrund der räumlichen Distanz notwendigerweise in Grenzen hielten. ${ }^{67}$

62 Noflatscher, Maximilian, 36.

63 RI XIV n. 10071 vom 8.4.1500; RI XIV n. 17707 vom 2.10.1503; Hochrinner, Bianca Maria, 88 f. zur Reise 1506.

64 Z. B. RI XIV nn. 9463 (16.10.1499), 13533 (10.7.1499), 14365 (1.9.1500), 14819 (9.1.1501).

65 Für Beispiele vgl. die Beiträge in Keller (Hg.), Gynäkokratie.

66 Zum Begriff Noflatscher, Maximilian, 40; zum Problem das Resümee bei Hollegger, Maximilian I., 258-270.

67 Überblick bei Tamussino, Margarethe; vgl. dazu LutTer, Repräsentation. 
Bianca hingegen war mit dem Problem konfrontiert, dass ihr Gemahl gleichzeitig anwesend und abwesend war. Der Mangel an Geld und „Ordnung“ bei gleichzeitigem Anspruch des Römischen Königs, für alle Agenden bis ins kleinste Detail selbst die Verantwortung zu übernehmen, machte es nahezu unmöglich, entweder eigenständige Aufgabenbereiche zu schaffen oder aber tatsächlich gemeinsam zu handeln. Damit hatten auch die männlichen Amtsträger des Königs zu kämpfen. Bianca hat bei der „Vertretung“ ihrer Familie gegenüber Frankreich besonders ab 1499 und auch in den Auseinandersetzungen gegen die Eidgenossen einige Versuche stellvertretenden Handelns unternommen - allein der Mangel an Kommunikation mit dem König und seinen Leuten macht ihr weitgehendes Scheitern in diesen Dingen eigentlich nicht verwunderlich. ${ }^{68}$ Vermutlich, so suggerieren jedenfalls viele Quellen, hätte sie sich ohnehin mit einem traditionellen Verständnis der Beteiligung an Maximilians Herrschaft im Rahmen seiner Herrschaftsrepräsentation und Klientelpolitik zufrieden gegeben, doch nicht einmal dafür scheint es mangels entsprechender Begegnungen viel Spielraum gegeben zu haben.

Man gewinnt den Eindruck, dass Bianca in einer besonders komplexen sozial-politischen Konstellation über besonders wenige handlungsleitende Modelle und Vorgaben verfügte, deren Nutzung es ihr erlaubt hätte, „erfolgreich“ zu agieren. Im Unterschied zu ihrer Stieftochter Margarethe fehlte ihr dafür neben den bereits genannten Faktoren im wörtlichen Sinn der Ort für ein solches Handeln. Stellte der burgundische Hof für Maria und Margarethe einen sozialen Raum zur Verfügung, der ihnen Vorbilder bot und ihre Lebensentwürfe tendenziell begünstigte, handelte es sich bei der höfischen Umgebung des Königs über weite Strecken um einen mobilen und kriegerischen Hof, um eine männlich dominierte Umgebung, die auf den Fürsten hin orientiert war. ${ }^{69}$ Auffallend ist im Vergleich mit dem burgundischen, aber auch dem savoyardischen Hof, wie wenig die Quellen über den Bildungshorizont der Königin und ihrer Umgebung berichten. So viel wir über Tafel- und Festkultur, höfisches Auftreten und standesgemäße Repräsentation bis in die Details von Kleidung, Schmuck und Toiletteartikeln erfahren, so wenig wissen wir bislang, ganz im Unterschied zu Maria oder Margarethe, über Biancas Erziehung und Ausbildung, Lektüregewohnheiten oder gar eine Bibliothek, die Auskunft über Rollenmodelle der Königin geben könnten.

Maximilians höfisches Umfeld bietet in seiner Widersprüchlichkeit zwischen ständischem und humanistischem Denken, provinziellem und europäischen Horizont, bürgerlichem und gelehrten Anspruch an sich schon ein ganz spezifisches Spannungsmoment. Es bleibt weiteren vergleichenden Forschungen vorbehalten, die Leerstelle, welche die zuletzt genannten Aspekte in diesem Zusammenhang noch markieren, zu füllen.

\section{Literatur}

Bojcov, Michail A., „Das Frauenzimmer“ oder „die Frau bei Hofe“?, in: Jan Hirschbiegel (Hg.), Das Frauenzimmer. Die Frau bei Hofe in Spätmittelalter und früher Neuzeit (= Residenzenforschung 11), Stuttgart 2000, 327-337.

Calvi, Felice, Bianca Maria Sforza-Visconti, regina dei romani, imperatrice germanica, e gli ambasciatori di Lodovico Moro alla corte cesarea secondo nuovi documenti, Milano 1888.

68 Vgl. z. B. RI XIV nn. 12975 und 12976 (beide 23.2.1499).

69 Vgl. Noflatscher, Maximilian, 33. 
Duby, Georges, [u. a.] (Hgg.), Geschichte der Frauen, 5 Bde., Frankfurt a. M. 1993-1995.

EICHBERGER, Dagmar (Hg.), Women of distinction. Margaret of York, Margaret of Austria (= Exibition Women of Distinction, Margaret of York and Margaret of Austria, Mechelen, Lamot, 17.9.-18.12.2005), Leuven 2005.

GATT, Anneliese, Der Innsbrucker Hof zur Zeit Kaiser Maximilians I. 1493-1519, Diss. Innsbruck 1943.

Griesebner, Andrea, Feministische Geschichtswissenschaft. Eine Einführung, Wien 2005.

Heinig, Paul-Joachim, Theorie und Praxis der „höfischen Ordnung“ unter Friedrich III. und Maximilian I., in: Holger Kruse/Werner Paravicini, Höfe und Hofordnungen 1200-1600 (= Residenzenforschung 10), Sigmaringen 1999, 223-242.

DERs., Umb merer zucht und ordnung willen. Ein Ordnungsentwurf für das Frauenzimmer des Innsbrucker Hofs aus den ersten Tagen Kaiser Karls V. (1519), in: Jan Hirschbiegel (Hg.), Das Frauenzimmer. Die Frau bei Hofe in Spätmittelalter und früher Neuzeit (= Residenzenforschung 11), Stuttgart 2000, 311-323.

Ders., Maximilian und die Frauen. In den Fängen der dynastischen Politik, in: Georg Schmidtvon Rhein (Hg.), Kaiser Maximilian I. Bewahrer und Reformer, Ramstein 2002, 69-81.

Hirschbiegel, Jan, Das Frauenzimmer. Die Frau bei Hofe in Spätmittelalter und früher Neuzeit (= Residenzenforschung 11), Stuttgart 2000.

Hochrinner, Heidemarie, Bianca Maria Sforza. Versuch einer Biographie, Diss. Graz 1966.

Hollegger, Manfred, Maximilian I. (1459-1519). Herrscher und Mensch einer Zeitenwende, Stuttgart 2005.

Johanek, Peter (Hg.), Adventus. Studien zum herrscherlichen Einzug in die Stadt (= Städteforschung A, 75), Köln-Wien 2009.

Keller, Kathrin (Hg.), Gynäkokratie. Frauen und Politik in der höfischen Gesellschaft der Frühen Neuzeit (= zeitenblicke 8, Nr. 2, http://www.zeitenblicke.de/2009/2/editorial/ index_html).

Kocher, Ursula/SIEber, Andrea (Hgg.), Maximilians Welt. Kaiser Maximilian I. im Spannungsfeld zwischen Innovation und Tradition (= Berliner Mittelalter- und Frühneuzeitforschung 11), Berlin 2011, im Druck.

Köfler Margarete/Caramelle, Silvia, Die beiden Frauen des Erzherzogs Sigmund von Österreich-Tirol (= Schlern-Schriften 269), Innsbruck 1982.

Kraus, Victor von, Maximilian's I. Beziehungen zu Sigmund von Tirol in den Jahren 14901496, Wien 1879.

Kruse, Holger/Paravicini, Werner (Hgg.), Höfe und Hofordnungen 1200-1600 (= Residenzenforschung 10), Sigmaringen 1999.

Le Glay, M. (Hg.), Correspondance de l'Empereur Maximilien Ier et de Marguerite d'Autriche, sa fille, gouvernante des Pays-Bas, de 1507 à 1519. Paris 1839 (repr. 1966).

Lutter, Christina, Politische Kommunikation in der Frühen Neuzeit am Beispiel der diplomatischen Beziehungen zwischen der Republik Venedig und Maximilian I. (1495-1508) (= Veröffentlichungen des Instituts für Österreichische Geschichtsforschung 34), Wien 1998.

DiEs., Zur Repräsentation von Geschlechterverhältnissen im höfischen Umfeld Maximilians, in: Ursula Kocher/Andrea Sieber (Hgg.), Maximilians Welt. Kaiser Maximilian I. im Spannungsfeld zwischen Innovation und Tradition (= Berliner Mittelalter- und Frühneuzeitforschung), Berlin 2011, im Druck. 
Middell Matthias/Sammler, Stefan (Hgg.), Alles Gewordene hat Geschichte. Die Schule der „Annales“ in ihren Texten 1929-1992, Leipzig 1994.

Müller, Jan-Dirk, Gedechtnus. Literatur und Hofgesellschaft um Maximilian I., München 1982.

Niederkorn, Christine, Der Hof Maximilians I. und das höfische Leben. Ein Beitrag zur höfischen Kulturgeschichte, Diss. Graz 1985.

Noflatscher, Heinz, Räte und Herrscher. Politische Eliten an den Habsburgerhöfen der österreichischen Länder 1480-1530 (= Veröffentlichungen des Instituts für Europäische Geschichte 161), Mainz 1999.

Ders., „Die Heuser Österreich vnd Burgund“. Zu den Quellen der Habsburgerhöfe um 1500 oder zu einem historiographischen Stresssyndrom, in: Frühneuzeit-Info 12/2 (2001), 3248.

Ders., Maximilian im Kreis der Habsburger, in: Georg Schmidt-von Rhein (Hg.), Kaiser Maximilian I. Bewahrer und Reformer, Ramstein 2002, 31-48.

Ders./Niederkorn, Jan Paul (Hgg.), Der Innsbrucker Hof. Residenz und höfische Gesellschaft in Tirol vom 15. bis 19 Jahrhundert (= Archiv für österreichische Geschichte 138), Wien 2005.

OpITz, Claudia, Um-Ordnungen der Geschlechter. Einführung in die Geschlechtergeschichte, Tübingen 2005.

Paravicini, Werner (Hg.), Alltag bei Hofe (= Residenzenforschung 5), Sigmaringen 1995.

RI XIV 1-4 = Ausgewählte Regesten des Kaiserreiches unter Maximilian I. 1493-1519, bisher 4 Bde., bearb. von Hermann Wiesflecker [u. a.] (J. F. Böhmer, Regesta Imperii XIV, 1-4), Wien-Köln-Weimar 1990-2007 [online siehe auch: www.regesta-imperii.de].

Rogge, Jörg, Nur verkaufte Töchter? Überlegungen zu Aufgaben, Quellen, Methoden und Perspektiven einer Sozial- und Kulturgeschichte hochadeliger Frauen und Fürstinnen im deutschen Reich während des späten Mittelalters und am Beginn der Neuzeit, in: Cordula Nolte et al. (Hgg.), Principes. Dynastien und Höfe im späten Mittelalter (= Residenzenforschung 14), Stuttgart 2002, 235-276.

SAnudo, Marino, I Diarii, 58 Bde., hg. von Guglielmo Berchet/Niccolò Barozzi/Marco Allegri, Venezia 1879-1902.

Schmidt-von Rhein, Georg (Hg.), Kaiser Maximilian I. Bewahrer und Reformer, Ramstein 2002.

Schnettger, Matthias, Weibliche Herrschaft in der Frühen Neuzeit. Einige Beobachtungen aus verfassungs- und politikgeschichtlicher Sicht, in: KELlER, Kathrin (Hg.), Gynäkokratie. Frauen und Politik in der höfischen Gesellschaft der Frühen Neuzeit (= zeitenblicke 8, Nr. 2, URL: http://www.zeitenblicke.de/2009/2/editorial/index_html).

Scoтt, Joan, Gender. A Useful Category of Historical Analysis, in: American Historical Review 91 (1986), 1053-1075.

SpIEss, Karl-Heinz, Unterwegs zu einem fremden Ehemann. Brautfahrt und Ehe in europäischen Fürstenhäusern des Spätmittelalters, in: Irene Erfen/Ders. (Hgg.); Fremdheit und Reisen im Mittelalter, Stuttgart 1997, 17-36.

Stollberg-Rilinger, Barbara (Hg.), Was heißt Kulturgeschichte des Politischen? (= Zeitschrift für Historische Forschung, Beiheft 35), Berlin 2005.

Tamussino, Ursula, Margarete von Österreich - Diplomatin der Renaissance, Graz-Wien 1995. 
WeIss, Sabine: Die vergessene Kaiserin. Bianca Maria Sforza. Kaiser Maximilians zweite Gemahlin, Innsbruck-Wien 2010.

WiESFLECKER, Angelika, Die „oberösterreichischen“ Kammerraitbücher zu Innsbruck: 14931519. Ein Beitrag zur Wirtschafts-, Finanz- und Kulturgeschichte der oberösterreichischen Ländergruppe, Graz 1987.

WiESFLECKer, Hermann, Maximilian I, das Reich, Österreich und Europa an der Wende zur Neuzeit, 5 Bde., Wien 1971-1986.

Wunder, Heide, „Er ist die Sonn', sie ist der Mond“. Frauen in der Frühen Neuzeit, München 1992.

DiEs., Herrschaft und öffentliches Handeln von Frauen in der Gesellschaft der Frühen Neuzeit, in: Ute Gerhard (Hg.), Frauen in der Geschichte des Rechts. Von der Frühen Neuzeit bis zur Gegenwart, München 1997.

Dies. (Hg.), Dynastie und Herrschaftssicherung in der Frühen Neuzeit. Geschlechter und Geschlecht (= Zeitschrift für Historische Forschung, Beiheft 28), Berlin 2002.

\begin{abstract}
While gender history has developed into a powerful branch of early modern history, we know surprisingly little about gender roles and relations in the courtly environment of Maximilian I, despite his comprehensive dynastic politics and two marriages. One reason might be that previous research concentrated on the individual personality of the emperor, as well as the specific personal constellations of Maximilian's relationships with Mary of Burgundy and his second wife, Bianca Maria Sforza. This article focuses on Bianca Maria starting from the observation that both her contemporaries and few biographers seem to have perceived her largely in terms of a failed dynastic project. What are the foundations of this perception, and might they be differentiated by gender related questions? How do seemingly "individual" qualities, behaviour, and actions relate to representations of gender roles, political motives and cultural traditions?
\end{abstract}

